Case
Reports

Aziz Ur Rehman, MD Abdelkader Almanfi, MD, MRCP-UK

Srikanth Nadella, MD Umair Sohail, MD
Key words: Celiac artery/ pathology/radiography; tomography, x-ray computed/methods; von Willebrand disease

From: Department of Internal Medicine, St. Luke's Hospital, Chesterfield, Missouri 63017

Address for reprints: Abdelkader Almanfi, MD MRCP-UK, Department of Cardiology, Texas Heart Institute, 6720 Bertner Ave., Houston, TX 77030

E-mail: aalmanfi@ stlukeshealth.org

(C) 2014 by the Texas Heart ${ }^{\circledR}$ Institute, Houston

\section{Isolated Spontaneous Celiac Artery Dissection}

\author{
in a 47-Year-Old Man with von Willebrand Disease
}

Isolated spontaneous dissection of the celiac artery is rare, and its occurrence without aortic dissection is even rarer. The typical symptom of this dissection is acute-onset abdominal pain. Complications of the condition include aneurysm formation, rupture, and abdominal-organ ischemia or infarction, especially in the liver or spleen. We report the case of a 47-year-old man with von Willebrand disease who had an isolated spontaneous dissection of the celiac artery. We used computed tomography and computed tomographic angiography in the diagnosis and characterization of the dissection. To our knowledge, this is the first report of celiac artery dissection in a patient with von Willebrand disease. (Tex

Heart Inst J 2014;41(3):344-5)

solated spontaneous dissection of the celiac artery is reportedly rare, and even rarer when unaccompanied by aortic dissection. We report the finding of an isolated spontaneous dissection of the celiac artery in a patient with von Willebrand disease, and our diagnostic use of computed tomography (CT) and CT angiography in this case.

\section{Case Report}

In March 2011, a 47-year-old man was admitted to our hospital because of persistent, dull pain in the upper left quadrant of the abdomen. The pain started after the patient ate, and it gradually worsened. About a week before admission, he had sharp epigastric pain that radiated to his back and lasted for approximately 10 minutes. He reported no fever, nausea, vomiting, rectal bleeding, melena, or dysuria. He had von Willebrand disease, upper gastrointestinal bleeding secondary to nonsteroidal drug use, trigeminal neuralgia, and no history of iatrogenic injury from instrumentation or trauma.

The patient's vital signs were stable. Abdominal examination revealed left upper quadrant tenderness without guarding or rigidity. Initial laboratory data included a hemoglobin level of $16.5 \mathrm{~g} / \mathrm{dL}$, a white blood cell count of 10,700 cells $/ \mathrm{mm}^{3}$ with a normal differential count, a C-reactive protein level of $4.3 \mathrm{mg} / \mathrm{L}$, an erythrocyte sedimentation rate of $23 \mathrm{~mm} / \mathrm{hr}$, and a normal partial thromboplastin time and prothrombin time. Further results were negative for autoimmune disease or vacuities. Contrast-enhanced CT of the abdomen and pelvis revealed an infarct in the posterior third of the spleen (Fig. 1), an opacified segmental branch of a splenic artery, and an eccentric thrombus and focal dissection involving the celiac artery with surrounding fat-stranding (Fig. 2). The dissection was also seen in the common hepatic artery (CHA). Computed tomographic angiograms revealed a normal aorta and an intimal flap within the celiac artery that extended into the splenic artery and the CHA, with small areas of thrombus along the left lateral wall of the celiac artery and the posterior wall of the CHA. The patient was discharged from the hospital on low-dose aspirin therapy, with follow-up CT angiography scheduled in 3 months.

\section{Discussion}

Contrast-enhanced CT is the primary technique for diagnosing celiac artery dissection; magnetic resonance imaging, magnetic resonance angiography, Doppler ultrasonography, and conventional angiography can also be used. On contrast-enhanced CT, findings might include an intimal flap, mural thrombus with or without aneurysmal dilation, segmental stenosis, and infiltration of the fat surrounding the vessel.' 


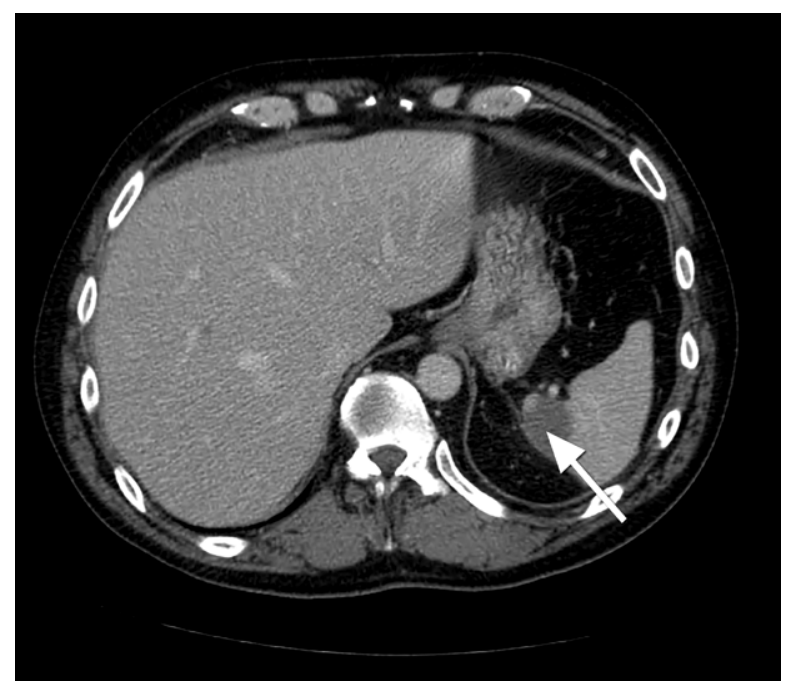

Fig. 1 Computed tomogram of the abdomen shows a splenic infarct (arrow) in the posterior third of the spleen.

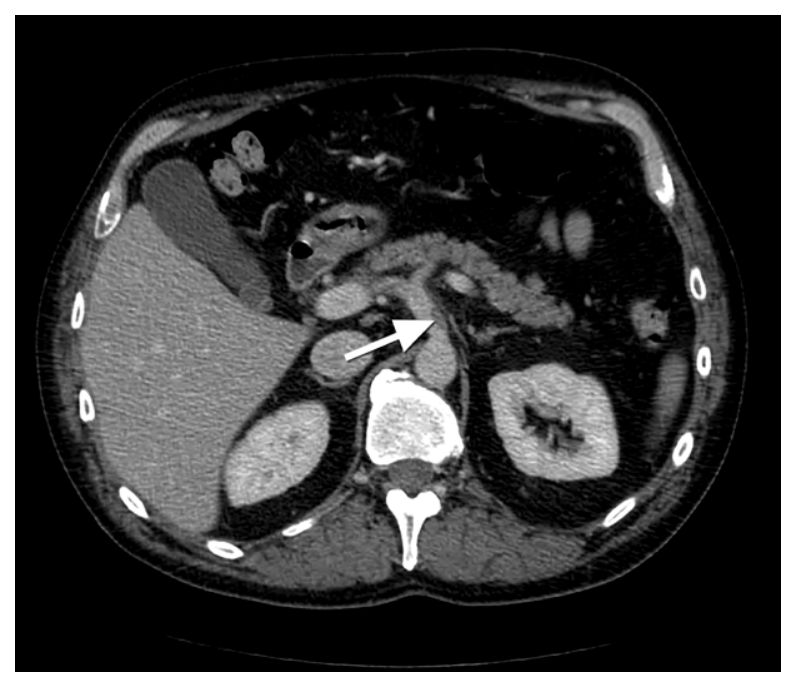

Fig. 2 Computed tomogram of the abdomen shows celiac artery dissection (arrow).

Several treatment options exist; however, no guidelines have been established for the optimal management of isolated spontaneous dissection of the celiac trunk. The prognosis depends on the degree of dissection and on whether the dissection extends into adjacent branches and causes organ ischemia.

Therapy should include anticoagulant or antiplatelet agents for 3 to 6 months, to prevent thromboembolic complications (targeted international normalized ratio, 2.0-3.0); and strict blood pressure control, to prevent progression of the dissection plane. The modification of cardiac risk factors limits the dissection's propagation and reduces the risk of rupture. ${ }^{2}$ Our patient's von Willebrand disease complicated his treatment, because that disease increases the risk of bleeding during anti- coagulant or antiplatelet use. After discussion with the hematologist, gastroenterologist, and patient, we prescribed low-dose aspirin and another CT angiogram in 3 months.

Surgical or endovascular treatment should be reserved for patients who remain symptomatic despite medical therapy and for patients whose disease is complicated by aneurysm, ischemia, or rupture. ${ }^{3}$ Endovascular treatment is an option for patients who are at high surgical risk, although its safety and effectiveness in comparison with surgery are unclear. ${ }^{3}$ To our knowledge, this is the first report of celiac artery dissection in a patient with von Willebrand disease.

\section{Acknowledgments}

The authors thank Diana Kirkland for editing the manuscript and James Philpot for preparing the figures.

\section{References}

1. D’Ambrosio N, Friedman B, Siegel D, Katz D, Newatia A, Hines J. Spontaneous isolated dissection of the celiac artery: CT findings in adults. AJR Am J Roentgenol 2007;188(6): W506-11.

2. Kim JH, Roh BS, Lee YH, Choi SS, So BJ. Isolated spontaneous dissection of the superior mesenteric artery: percutaneous stent placement in two patients. Korean J Radiol 2004;5(2): 134-8.

3. Fenoglio L, Allione A, Scalabrino E, Alberto G, Benedetti V, Pomero F, et al. Spontaneous dissection of the celiac artery: a pitfall in the diagnosis of acute abdominal pain. Presentation of two cases. Dig Dis Sci 2004;49(7-8):1223-7. 